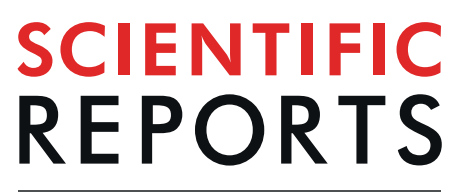

natureresearch

\title{
Nectin-4 cis-interacts with ErbB2 and its trastuzumab-resistant splice variants, enhancing their activation and DNA synthesis
}

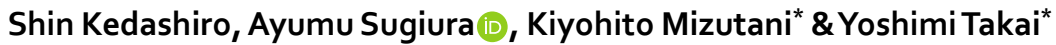

Nectin-4 cell adhesion molecule and ErbB2 tyrosine kinase receptor are upregulated in many cancers, including breast cancer, and promote cancer cell proliferation and metastasis. Using human breast cancer cell lines T47D and SUM190-PT, in which both nectin-4 and ErbB2 were upregulated, we showed here that nectin-4 cis-interacted with ErB2 and enhanced its dimerization and activation, followed by the activation of the phosphoinositide 3-kinase-AKT signalling pathway for DNA synthesis. The third immunoglobulin-like domain of nectin-4 cis-interacted with domain IV of ErbB2. This region differs from the trastuzumab-interacting region but is included in the trastuzumab-resistant splice variants of ErbB2, p95-ErbB2 and ErbB2 $\Delta$ Ex16. Nectin-4 also cis-interacted with these trastuzumab-resistant splice variants and enhanced the activation of the phosphoinositide 3-kinase-AKT signalling pathway for DNA synthesis. In addition, nectin-4 enhanced the activation of the p95-ErbB2-induced JAK-STAT3 signalling pathway, but not the ErbB2- or ErbB2 $\Delta$ Ex16-induced JAK-STAT3 signalling pathway. These results indicate that nectin-4 cis-interacts with ErbB2 and its trastuzumab-resistant splice variants and enhances the activation of these receptors and downstream signalling pathways in a novel mechanism.
\end{abstract}

ErbB2 is a receptor tyrosine kinase belonging to the epidermal growth factor (EGF) receptor family with four members, ErbB1, -2, -3, -4, which are also known as EGF receptor for ErbB1 and HER1, -2, -3, -4, respectively ${ }^{1}$. ErbB2 is frequently upregulated by its gene amplification in many types of cancers, including breast cancer, and plays a role as an oncogenic protein, which induces tumourigenesis, invasion, and metastasis ${ }^{1-5}$. The incidence of ErbB2-upregulated breast cancers is approximately $20-30 \%$ of total breast cancer cases ${ }^{6,7}$. ErbB2-targeting monoclonal antibody (mAb) drugs, such as trastuzumab (also known as Herceptin) and pertuzumab (also known as Perjeta), have been developed and used as therapeutic drugs for ErbB2-upregulated breast cancers ${ }^{8}$. However, two splice variants of ErbB2, p95-ErbB2 and ErbB2 $\Delta$ Ex16, have been identified as oncogenic proteins in trastuzumab-resistant breast cancers ${ }^{9-20}$, although trastuzumab-resistance to ErbB2 $\Delta$ Ex16 is controversial ${ }^{21,22}$. The incidence of p95-ErbB2- or ErbB2 $\Delta$ Ex16-upregulated breast cancers is $30 \%$ and $90 \%$, respectively, of the total ErbB2-upregulated breast cancers ${ }^{14,23,24}$. Thus, the development of therapeutic drugs for trastuzumab-resistant breast cancers expressing such variants is awaited.

Of the ErbB family members, the activation mechanism has most extensively been investigated for ErbB $1^{25}$. One EGF molecule binds to one ErbB1 molecule, resulting in its homodimerization. Crystal structure and NMR analyses have revealed that the ErbB1 molecule exists in a 'tethered' or an 'extended' structure in the absence of $\mathrm{EGF}^{25,26}$. In the tethered structure, the extracellular domains II and IV are linked, but in the extended structure of ErbB1, domain II, which is a binding site for another ErbB1 molecule, is released ${ }^{25}$ (Fig. 1a). These two structures are in dynamic equilibrium, and the binding of one EGF molecule to domain I and domain III promotes the conversion of the tethered structure to the extended structure (Fig. 1a). This conversion leads to the homodimerization of ErbB1, promoting the tyrosine kinase activity of each receptor, followed by the intermolecular tyrosine phosphorylation (Fig. 1a). The Src homology 2 domain-containing protein Grb2 binds to the tyrosine-phosphorylated ErbB1 molecule and activates at least three signalling pathways, Ras-Raf-MEK-ERK, phosphoinositide 3-kinase (PI3K)-AKT, and JAK-STAT signalling pathways, for cell proliferation, survival, and

From the Division of Pathogenetic Signaling, Department of Biochemistry and Molecular Biology, Kobe University Graduate School of Medicine, 1-5-6 Minatojima-minamimachi, Chuo-ku, Kobe, Hyogo, 650-0047, Japan. *email: mizutani@med.kobe-u.ac.jp; ytakai@med.kobe-u.ac.jp 
a
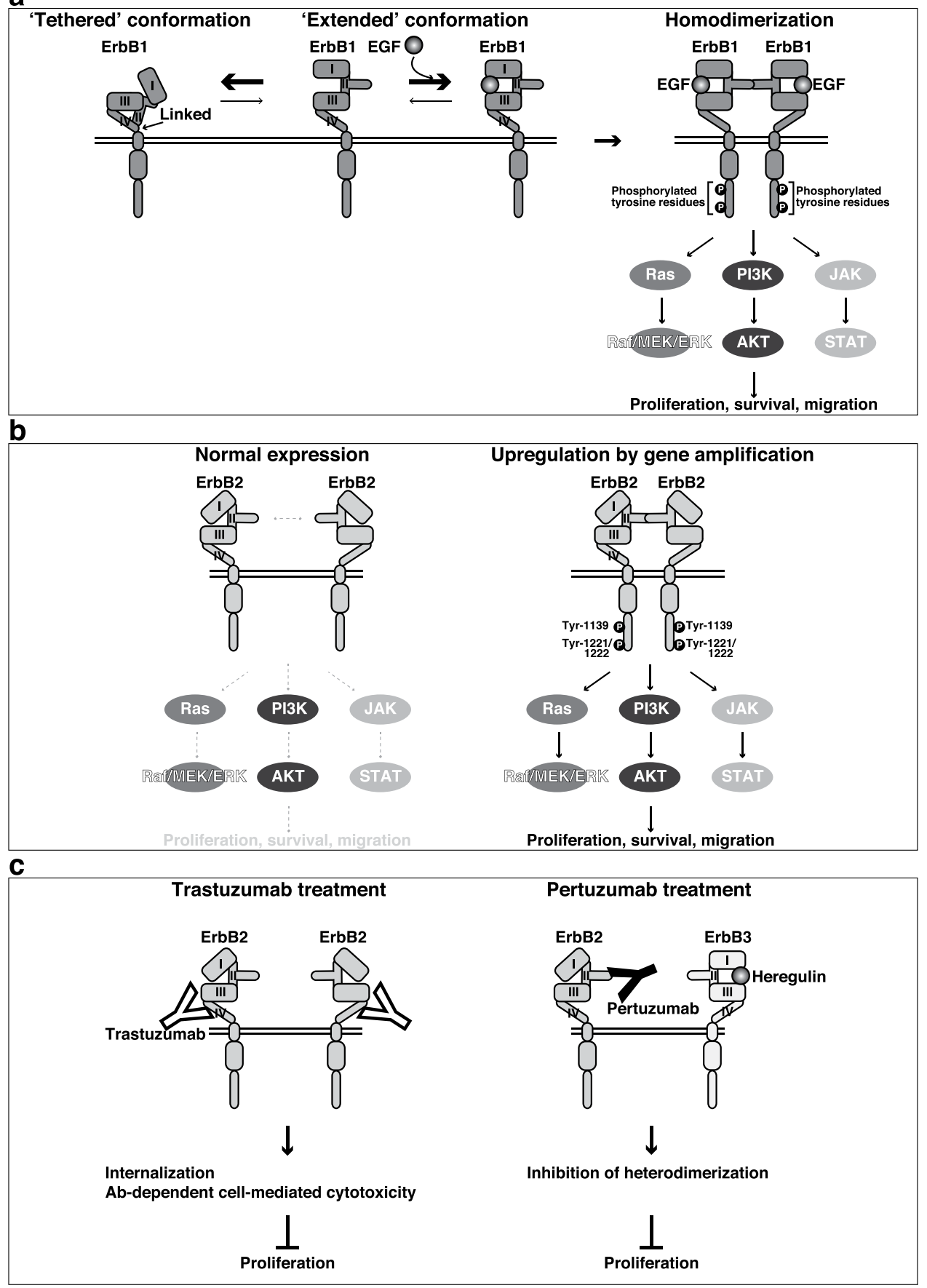

Figure 1. Schematic presentation of the mechanism for the homodimerization and activation of ErbB1 and ErbB2 and the inhibitory mechanism for the activation of ErbB2 by its antibodies. (a) A mechanism for ErbB1. (b) A mechanism for ErbB2. (c) Inhibitory mechanism for the activation of ErbB2 by trastuzumab and pertuzumab.

migration, and their continuous abnormal activation eventually leads to cancer cell tumourigenesis, invasiveness, and metastasis ${ }^{1-5,27-32}$. The truncated mutant of ErbB1 is an oncogenic protein that possesses the homodimerization property even in the absence of EGF and continuously transduces cell proliferation signals ${ }^{33,34}$.

ErbB2 has a kinase domain in the cytoplasmic tail, but any ligand for ErbB2 has not been identified, unlike ErbB1, ErbB3, and ErbB4, which have specific ligands ${ }^{1-5}$. Crystallographic studies have revealed that ErbB2 exists in a distinct structure in which it exhibits an extended structure in the absence of ligands so that the loop in domain II of one ErbB2 molecule is ready to bind to that of another ErbB2 moolecule ${ }^{35}$ (Fig. 1b). However, the affinity of the loop in domain II of ErbB2 for its homodimerization is low and the homodimerization of ErbB2 requires its upregulation, which is frequently induced by amplification of ERBB2 in various cancers, including breast cancer ${ }^{31}$. The upregulated ErbB2-induced homodimerization enhances its tyrosine-phosphorylation and 
the activation of at least the same three signalling pathways as those downstream of ErbB1 for cell proliferation, survival, and migration, eventually leading to cancer cell tumourigenesis, invasiveness, and metastasis ${ }^{1-5,27-32}$ (Fig. 1b). Thus, upregulated ErbB2 also serves as an oncogenic protein in this mechanism. Trastuzumab interacts with domain IV of ErbB2 and enhances its internalization, causing inhibition of the ErbB2 signalling pathway for cell proliferation, although its mode of action differs depending on cancer cell type ${ }^{18}$ (Fig. 1c). Trastuzumab further targets tumour cells by antibody $(\mathrm{Ab})$-dependent cell-mediated cytotoxicity in a patient's immune system. Pertuzumab interacts with domain II of ErbB2 and inhibits its heterodimerization with ErbB3 and activation, causing inhibition of the ErbB2 signalling pathway for cell proliferation ${ }^{36,37}$ (Fig. 1c).

Nectin- 4 is a cell adhesion molecule (CAM), which was originally identified by Lopez's group ${ }^{38}$. It belongs to the nectin-like molecule (Necl) family with five members (Necl-1, -2, -3, -4 and -5), which comprises a superfamily with the nectin family with four members (nectin-1, $-2,-3$, and -4$)^{39-41}$. These members trans-interact with each other in a homophilic or heterophilic manner and regulate diverse events mediated by cell-cell adhesion in various cells ${ }^{39-41}$. In addition, they cis-interact with cell surface membrane receptors, such as the platelet-derived growth factor receptor, the fibroblast growth factor receptor, the vascular endothelial growth factor receptor, the prolactin receptor, ErbB3, and ErbB4, and integrins, such as integrin $\alpha v \beta 3$ and integrin $\alpha 6 \beta 4$, and regulate not only cell-cell adhesion but also cell migration, proliferation, differentiation, and survival ${ }^{42-45}$.

Nectin- 4 is upregulated in various cancers, such as breast ${ }^{46}$, lung $^{47}$, ovarian ${ }^{48}$, pancreatic ${ }^{49}$, gallbladder ${ }^{50}$, and gastric cancer ${ }^{51}$, and promotes cancer cell proliferation and metastasis ${ }^{47,50,52,53}$. The PI3K-AKT signalling pathway is implicated in these roles of nectin- 4 through activation of the Wnt- $\beta$-catenin and Rac small G protein signalling pathways ${ }^{51,54}$. In addition, breast cancer cells cultured in soft agar express nectin- 4 and nectin- 1 that trans-interact each other in a heterophilic manner, and nectin- 4 also trans-interacts in a homophilic manner ${ }^{52}$. These interactions enable the cancer cells to survive in an anchorage-independent manner in soft agar ${ }^{52}$. Nectin- 4 further enables epithelial and cancer cells to resist ferroptosis by clustering each cell for survival under a matrix-detached condition $^{55}$. The concept derived from these results for anti-cancer drugs and a diagnostic marker development by interception of nectin- 4 function has now been applied ${ }^{46,56-58}$.

We found here that nectin-4 cis-interacted with ErbB2 and enhanced its homodimerization and activation in a novel mechanism, followed by the activation of the PI3K-AKT signalling pathway for DNA synthesis. We further found that nectin-4 also cis-interacted with the trastuzumab-resistant splice variant of ErbB2, p95-ErbB2, and a more aggressive splice variant for tumourigenesis, ErbB2 $\Delta$ Ex16, and enhanced the activation of the PI3K-AKT signalling pathway for DNA synthesis. In addition, nectin-4 enhanced the activation of the p95-ErbB2-induced JAK-STAT3 signalling pathway, but not the ErbB2- or ErbB2 $\triangle$ Ex16-induced JAK-STAT3 signalling pathway.

\section{Results}

Cis-interaction of nectin-4 with ErbB2. We first examined whether nectin-4 cis-interacts with ErbB2. FLAG-tagged nectin-4 (FLAG-Nectin-4) was co-expressed with GFP-tagged ErbB2 (ErbB2-GFP) in human embryonic kidney (HEK) 293E cells. The cells were cultured in suspension to enable the detection of a possible cis-interaction between nectin- 4 and ErbB2 on the same plasma membrane. When FLAG-Nectin- 4 was immunoprecipitated using an anti-FLAG mAb, ErbB2-GFP was co-immunoprecipitated with FLAG-Nectin-4 (Fig. 2a). ErbB1-GFP, ErbB3-GFP, or ErbB4-GFP was not co-immunoprecipitated with FLAG-Nectin-4 under the same assay condition (Supplementary Fig. 1b-e). When endogenous nectin-4 was immunoprecipitated using an anti-nectin-4 polyclonal Ab (pAb) in SUM190-PT breast cancer cells, endogenous ErbB2 was co-immunoprecipitated with endogenous nectin-4 (Fig. 2b). In this cell line, nectin- 4 and nectin-1 were expressed, but nectin-2, nectin-3, Necl-1, Necl-2, Necl-3, Necl-4, or Necl-5, was not detected (Supplementary Fig. 1a). ErbB1, ErbB2, ErbB3, and ErbB4 were also expressed. In addition, endogenous nectin-4 and ErbB2 were co-localized at cellcell adhesion sites in T47D and SUM190-PT cells (Supplementary Fig. 1f). These results indicate that nectin-4 cis-interacts specifically with ErbB2 among the ErbB family members and that this cis-interaction occurs on the same plasma membrane.

Enhancement of the homodimerization and tyrosine-phosphorylation of ErbB2 by nectin-4. We then examined whether nectin-4 affects the homodimerization of ErbB2. HA-tagged ErbB2 (ErbB2-HA) and ErbB2-GFP were co-expressed with FLAG-Nectin-4 or with FLAG as control in HEK293E cells. When ErbB2-GFP was immunoprecipitated with an anti-GFP pAb, ErbB2-HA was co-immunoprecipitated with ErbB2-GFP and FLAG-Nectin-4, but not FLAG, and the amount of ErbB2-HA in the cells co-expressing FLAG-Nectin-4 was more than that co-immunoprecipitated with ErbB2-GFP in the cells co-expressing FLAG (Fig. 3a). These results indicate that nectin-4 cis-interacts with ErbB2 and that this cis-interaction enhances its homodimerization.

The homodimerization of ErbB2 induces the tyrosine-phosphorylation of ErbB2 intermolecularly at several tyrosine residues including 1139,1221 , and $1222^{59-61}$. Using mAbs, one of which recognizes phosphorylated tyrosine residue at 1139 and the other of which recognizes both phosphorylated tyrosine residues at 1221 and 1222, we examined whether the nectin-4-enhanced homodimerization of ErbB2 enhances the phosphorylation of these tyrosine residues. For this purpose, we used T47D breast cancer cells, which expressed both nectin- 4 and ErbB2 at much lower levels than SUM190-PT cells (Supplementary Fig. 1a). In this cell line, nectin-1 and Necl2, but not nectin-2, nectin-3, Necl-1, Necl-3, Necl-4, or Necl-5, were detected. The phosphorylation of tyrosine residues at 1139,1221 , and 1222 was enhanced in the T47D cells stably expressing FLAG-Nectin- 4 compared with that in the control cells (Fig. 3b). Conversely, the phosphorylation of these tyrosine residues was reduced by the siRNA-induced knockdown of NECTIN4 in SUM190-PT cells (Fig. 3c). The reduction of nectin-4 by the siRNA-induced knockdown was confirmed by Western blotting (Fig. 3c). These results indicate that nectin-4 enhances the homodimerization of ErbB2, which leads to the phosphorylation of its tyrosine residues at 1139, 1221 , and 1222 . 
a

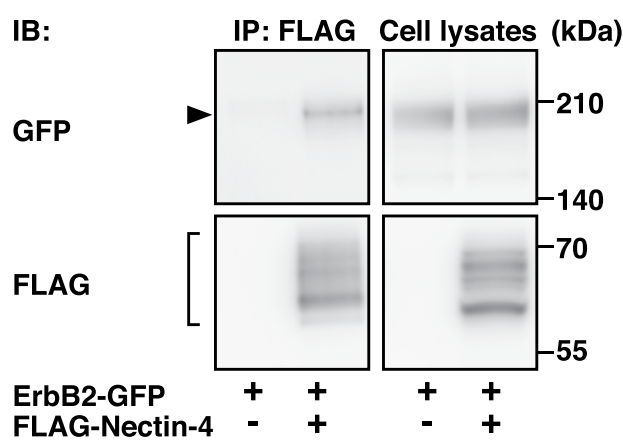

b

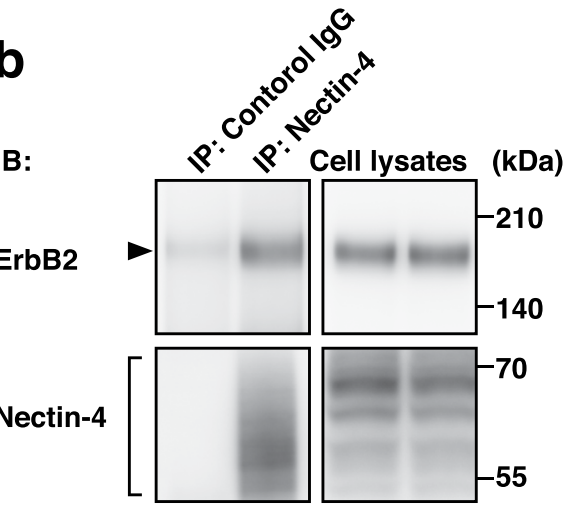

Figure 2. Cis-interaction of nectin- 4 with ErbB2. (a) Cis-interaction of nectin- 4 with ErbB2. HEK293E cells were co-transfected with various combinations of the indicated plasmids and cultured. The cells were detached using Accutase and cultured for $1 \mathrm{~h}$ in suspension. The cells were collected and lysed, and FLAG-tagged nectin-4 (FLAG-Nectin-4) was immunoprecipitated using an anti-FLAG mAb. The samples were subjected to Western blotting using the indicated Abs. (b) Cis-interaction of endogenous nectin- 4 with endogenous ErbB2. SUM190-PT cells were detached using Accutase and cultured for $1 \mathrm{~h}$ in suspension. The cells were collected and lysed, and endogenous nectin- 4 was immunoprecipitated using an anti-nectin- $4 \mathrm{pAb}$. The samples were subjected to Western blotting using the indicated Abs. Arrowheads and square brackets indicate each of the proteins. The displayed blots were cropped, and the full-length blots are shown in Supplementary Fig. 4. IB, immunoblotting; IP, immunoprecipitation. Representative results from three independent experiments are shown.

Selective enhancement of the activation of the PI3K-AKT signalling pathway by nectin-4. The tyrosine-phosphorylation of ErbB2 leads to the activation of the PI3K-AKT, Ras-Raf-MEK-ERK, and JAK-STAT signalling pathways ${ }^{1-5,27-30,32}$. We therefore examined the effects of nectin- 4 on the activation of these signalling pathways. The threonine-phosphorylation of AKT was markedly enhanced in the T47D cells stably expressing FLAG-Nectin-4 compared with that in the control cells, whereas the threonine- and tyrosine-phosphorylation of ERK1/2 or the tyrosine-phosphorylation of STAT3 was not significantly enhanced in the T47D cells stably expressing FLAG-Nectin-4 compared with that in the control cells (Fig. 4a). The threonine-phosphorylation of AKT was inhibited by the tyrosine kinase inhibitor for ErbB2, irbinitinib, in the T47D cells stably expressing FLAG-Nectin-4 and in the control cells (Fig. 4b). Conversely, the threonine-phosphorylation of AKT was reduced in the SUM190-PT cells in which endogenous nectin-4 was knocked down compared with that in the control cells (Fig. 4c). These results indicate that nectin-4 mainly enhances the ErbB2-mediated PI3K-AKT signalling pathway, but not the Ras-Raf-MEK-ERK1/2 signalling pathway or the JAK-STAT3 signalling pathway.

Enhancement of DNA synthesis by nectin-4 through the ErbB2-mediated PI3K-AKT signalling pathway. The enhancement of the phosphorylation of tyrosine residues of ErbB2 induces cell proliferation through the PI3K-AKT, Ras-Raf-MEK-ERK, and JAK-STAT signalling pathways ${ }^{1-5,27-30,32}$. We estimated cell proliferation by measuring the incorporation of the DNA base analogue 5-ethynyl-2'-deoxyuridine (EdU) into DNA. We first examined whether nectin-4 enhances the ErbB2-mediated DNA synthesis. The incorporation of EdU into the DNA of the T47D cells stably expressing FLAG-Nectin-4 was enhanced compared with that in the control cells (Fig. 5a-c). The incorporation of EdU into the DNA of the T47D cells stably expressing FLAG-Nectin-4 and the control cells was inhibited by irbinitinib (Fig. 5i). In addition, the incorporation of EdU into the DNA of the SUM190-PT cells in which endogenous nectin-4 was knocked down was reduced compared with that in the control cells (Fig. 5d-h). These results indicate that nectin-4 enhances the ErbB2-mediated DNA synthesis.

We then examined which signalling pathway, PI3K-AKT, Ras-Raf-MEK-ERK1/2, and/or JAK-STAT3 signalling pathways, is involved in this nectin-4-enhanced ErbB2-mediated DNA synthesis. The incorporation of EdU into DNA in the T47D cells stably expressing FLAG-Nectin-4 and the control cells was inhibited by the PI3K inhibitors wortmannin and LY294002 and the MEK inhibitor U0126, but not by the JAK1/2 inhibitor ruxolitinib (Fig. 5i). These results indicate that nectin-4 enhances the ErbB2-mediated DNA synthesis through the activation of the PI3K-AKT signalling pathway, and that the nectin-4-independent ErbB2-mediated activation of the Ras-Raf-MEK-ERK1/2 pathway, but not the JAK-STAT3 pathway, is also involved in the ErbB2-mediated DNA synthesis.

The cis-interaction of the third immunoglobulin-like domain of nectin-4 with domain IV of ErbB2. We next attempted to determine the ErbB2-interacting domain of nectin-4. For this purpose, FLAG-tagged mutants of nectin- 4 in which the cytoplasmic domain was deleted (FLAG-Nectin4-immunoglobulin (Ig)1/2/3-transmembrane (TM)), the cytoplasmic domain and first Ig-like domains were deleted (FLAG-Nectin-4-Ig2/3-TM), the cytoplasmic domain and the first and second Ig-like domains were deleted (FLAG-Nectin-4-Ig3-TM), and the first, second, and third Ig-like domains were 
a

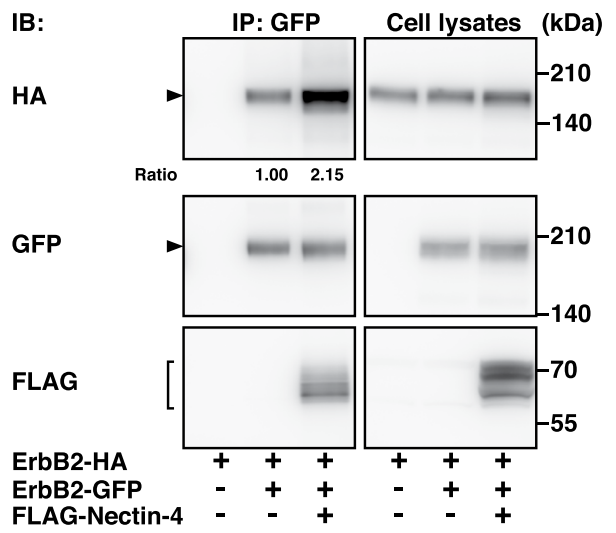

b

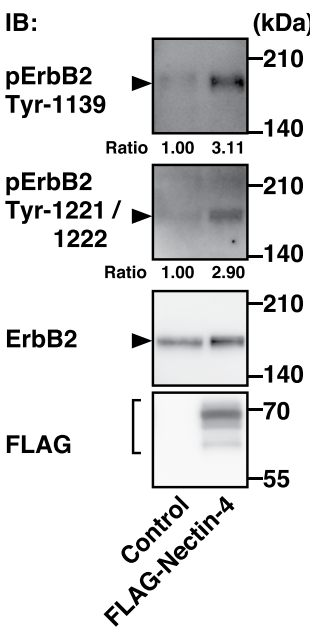

C

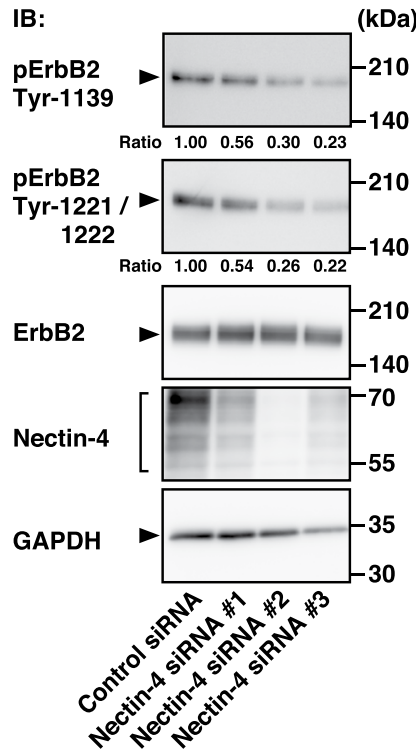

Figure 3. Enhancement of the homodimerization and tyrosine-phosphorylation of ErbB2 by nectin-4. (a) Enhancement of the homodimerization of ErbB2 by nectin-4. HEK293E cells were co-transfected with various combinations of the indicated plasmids, and the cells were detached using Accutase and cultured for $1 \mathrm{~h}$ in suspension. The cells were collected and lysed, and GFP-tagged ErbB2 (ErbB2-GFP) was immunoprecipitated using an anti-GFP pAb. The samples were subjected to Western blotting using the indicated Abs. Ratio represents the band intensities of the co-immunoprecipitaeted HA-tagged ErbB2 (ErbB2-HA) that were normalized to those of the immunoprecipitated ErbB2-GFP, and the normalized value of the both ErbB2-HA and ErbB2-GFP, but not FLAG-tagged nectin-4 (FLAG-Nectin-4), expressing cells was set as 1.00. (b) Enhancement of the tyrosine-phosphorylation of ErbB2 by nectin-4. T47D cells stably expressing FLAGNectin- 4 were serum-starved for $24 \mathrm{~h}$, and the samples were subjected to Western blotting using the indicated Abs. $\mathbf{c}$ Reduction of the tyrosine-phosphorylation of ErbB2 by knockdown of NECTIN4. SUM190-PT cells were transfected with a control siRNA or NECTIN4 siRNAs. The cells were serum-starved for $24 \mathrm{~h}$, and the samples were subjected to Western blotting using the indicated Abs. Ratio represents the band intensities of the phospho-ErbB2 on Tyr-1139 or Tyr-1221/1222 that were normalized to those of the total ErbB2, and the normalized value of the control cells was set as 1.00. Arrowheads and square brackets indicate each of the proteins. The displayed blots were cropped, and the full-length blots are shown in Supplementary Fig. 5. IB, immunoblotting; IP, immunoprecipitation. pErbB2, phospho-ErbB2. Representative results from three independent experiments are shown.

deleted (FLAG-Nectin-4-TM-cytoplasmic (CP)), were prepared (Supplementary Fig. 2a), and full-length FLAG-tagged or each of these nectin-4 mutants was co-expressed with ErbB2-GFP in HEK293E cells. When each of the FLAG-tagged nectin-4 mutants was immunoprecipitated using the anti-FLAG mAb, ErbB2-GFP was co-immunoprecipitated with full-length nectin-4 (FLAG-Nectin-4-FL), FLAG-Nectin-4-Ig1/2/3-TM, FLAG-Nectin-4-Ig2/3-TM, and FLAG-Nectin-4-Ig3-TM, but not with FLAG-Nectin-4-TM-CP (Supplementary Fig. 2b). These results indicate that nectin-4 cis-interacts with ErbB2 through the third Ig-like domain, although it cannot be excluded that other domains are also involved in the cis-interaction with ErbB2.

We then examined the nectin-4-interacting domain of ErbB2. For this purpose, GFP-tagged mutants of ErbB2, in which the cytoplasmic domain was deleted (ErbB2-1/2/3/4-TM-GFP), the cytoplasmic domain and domain I were deleted (ErbB2-2/3/4-TM-GFP), the cytoplasmic domain, domain I, and domain II were deleted (ErbB2-3/4-TM-GFP), and the cytoplasmic domain, domain I, domain II, and domain III were deleted (ErbB24-TM-GFP), were prepared (Supplementary Fig. 2a) and full-length GFP-tagged or each of these ErbB2 mutants was co-expressed with FLAG-Nectin-4 in HEK293E cells. When each of the GFP-tagged ErbB2 mutants was immunoprecipitated using the anti-GFP pAb, FLAG-Nectin-4 was co-immunoprecipitated with full-length ErbB2 (ErbB2-FL-GFP), ErbB2-1/2/3/4-TM-GFP, ErbB2-2/3/4-TM-GFP, ErbB2-3/4-TM-GFP, and ErbB24-TM-GFP (Supplementary Fig. 2c). Essentially the same results were obtained in the reciprocal immunoprecipitation assay (Supplementary Fig. 2d-h). These results indicate that ErbB2 cis-interacts with nectin-4 through domain IV, although it cannot be excluded that other domains are also involved in the cis-interaction with nectin-4.

Inhibition of the cis-interaction of nectin-4 with ErbB2 by the recombinant protein of the third Ig-like domain of nectin-4. We then examined whether a recombinant third Ig-like domain of nectin- 4 shows an inhibitory effect on the cis-interaction of full-length nectin- 4 with ErbB2 as schematically shown in Supplementary Fig. 3a-c, because this domain was a minimal interacting region with ErbB2. 


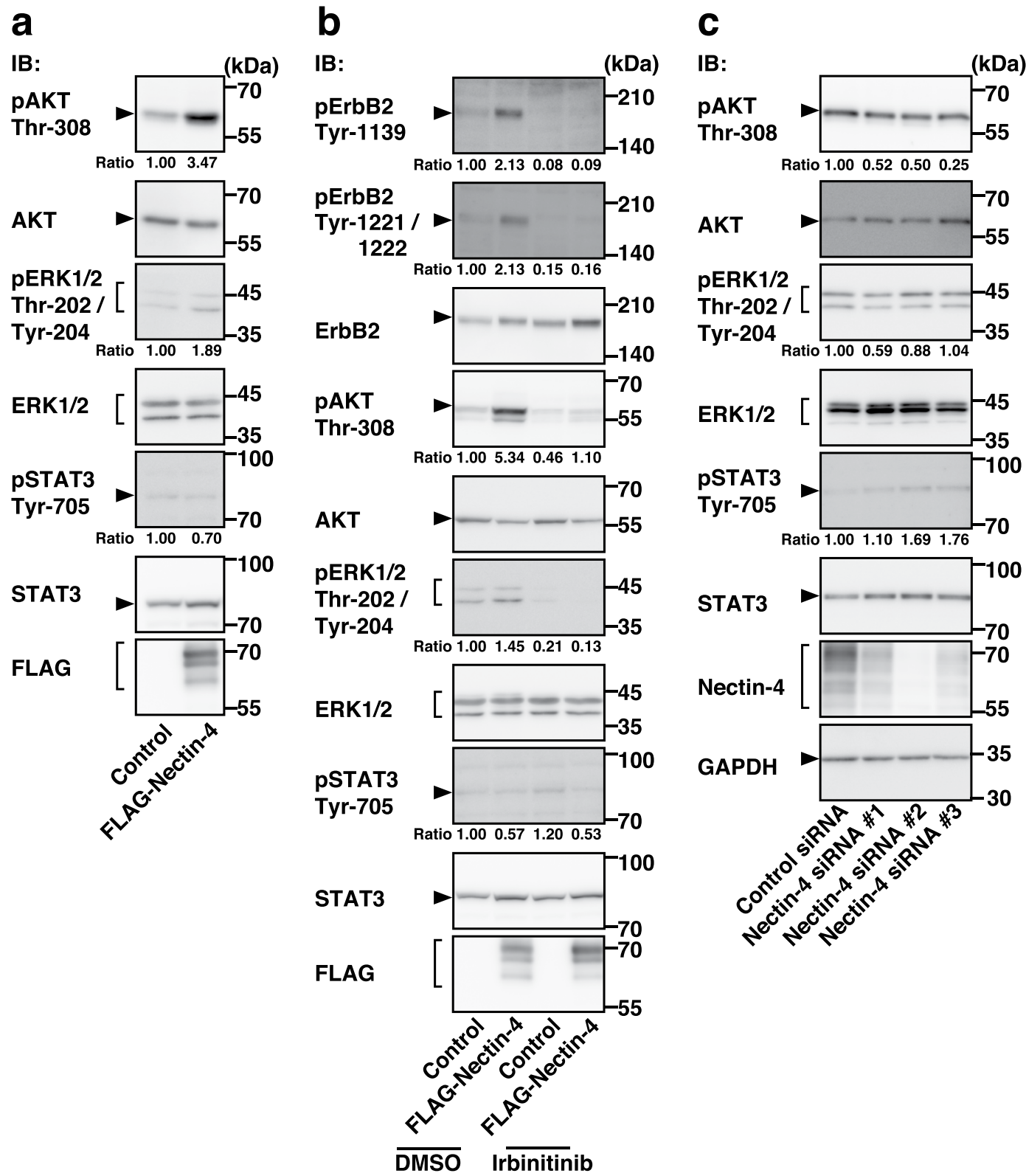

Figure 4. Selective enhancement of the activation of the PI3K-AKT signalling pathway by nectin-4. (a) Selective enhancement of the activation of the PI3K-AKT signalling pathway by nectin-4. T47D cells stably expressing FLAG-tagged nectin-4 (FLAG-Nectin-4) were serum-starved for $24 \mathrm{~h}$, and the samples were subjected to Western blotting using the indicated Abs. (b) Reduction of the threonine-phosphorylation of AKT and threonine- and tyrosine-phosphorylation of ERK1/2 by an ErbB2 inhibitor. T47D cells stably expressing FLAG-Nectin- 4 were serum-starved and treated with the ErbB2 inhibitor irbinitinib at $1 \mu \mathrm{M}$. The assay was carried out as in (a). (c) Reduction of the threonine-phosphorylation of AKT by the knockdown of NECTIN4. SUM190-PT cells were transfected with a control siRNA or NECTIN4 siRNAs. The assay was carried out as (a). Ratio represents the band intensities of each phosphorylated protein on the indicated tyrosine and threonine residues that were normalized to those of each total protein, and the normalized value of the control cells (DMSO-, but not Irbinitinib-, treated cells (b) or control siRNA cells (c)) was set as 1.00. Arrowheads and square brackets indicate each of the proteins. The displayed blots were cropped, and the full-length blots are shown in Supplementary Figs. 6 and 7. IB, immunoblotting; pAKT, phospho-AKT; pErbB2, phosphoErbB2; pERK1/2, phospho-ERK1/2; pSTAT3, phospho-STAT3. Representative results from three independent experiments are shown.

Recombinant FLAG-tagged extracellular region of nectin-4 (rec-FLAG-Nectin-4-Ig1/2/3) or recombinant FLAG-tagged third Ig-like domain of nectin-4 (rec-FLAG-Nectin-4-Ig3) was expressed in HEK293E cells and affinity-purified (Supplementary Fig. 3d). ErbB2-GFP was immunopurified from ErbB2-GFP-transfected HEK293E cells using anti-GFP-pAb-conjugated beads. When the fixed amount of rec-FLAG-Nectin-4-Ig1/2/3 

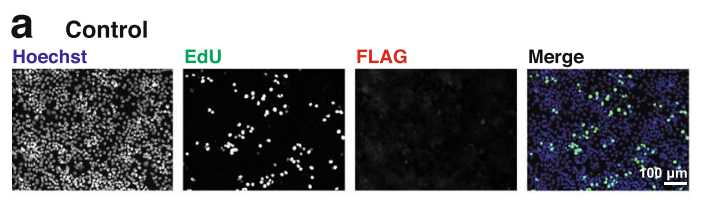

C
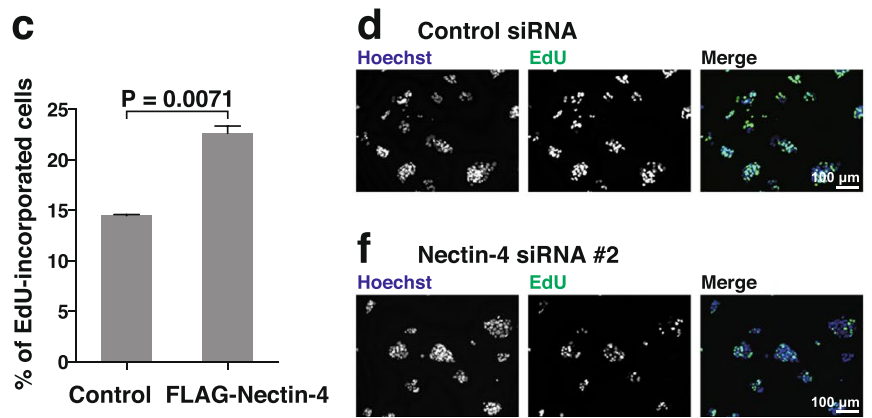
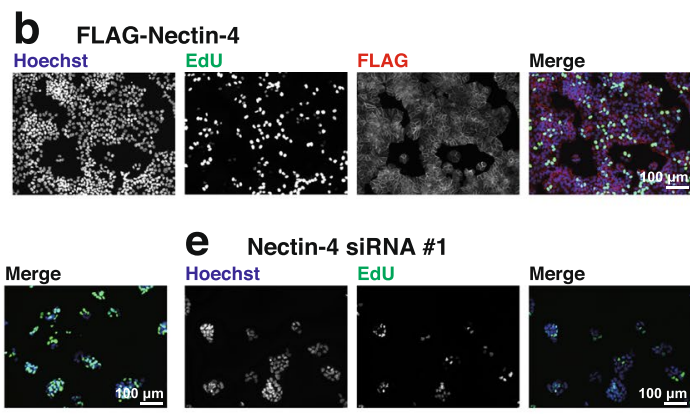

e Nectin-4 siRNA \#1
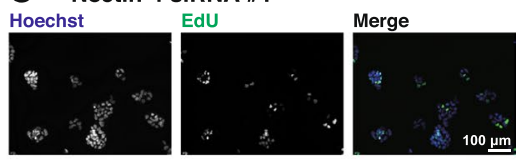

9 Nectin-4 siRNA \#3
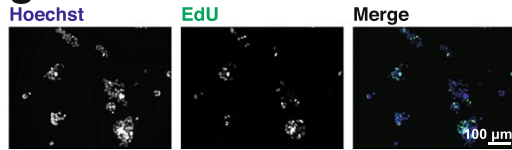
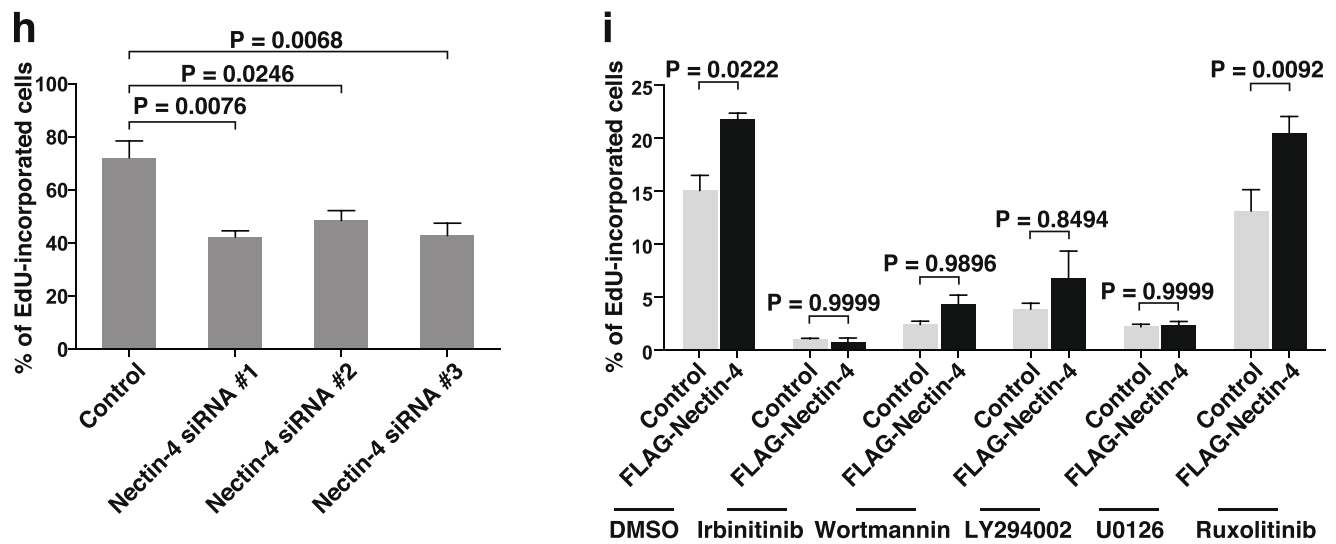

Figure 5. Enhancement of DNA synthesis by nectin-4 through the ErbB2-mediated PI3K-AKT signalling pathway. (a-c) Enhancement of DNA synthesis by nectin-4. T47D control cells (a) or T47D cells stably expressing FLAG-tagged nectin-4 (FLAG-Nectin-4) (b) were serum-starved and treated with medium containing $10 \mu \mathrm{M}$ EdU for $12 \mathrm{~h}$. After washout of the medium, the cells were cultured in the absence of serum and stained with an anti-DDDDK mAb, Hoechst33342, and EdU staining reagents. The number of EdUincorporated cells was counted by microscopy and Hybrid Cell Counter software (c). (d-h) Reduction of DNA synthesis by the knockdown of NECTIN4. SUM190-PT cells were transfected with a control siRNA (d) or NECTIN4 siRNAs (e-g), and the number of EdU-incorporated cells was counted by microscopy and Hybrid Cell Counter software (h). The assay was carried out as in (a-c). (i) Reduction of DNA synthesis by ErbB2, PI3K, and MEK inhibitors, but not a JAK inhibitor. T47D cells stably expressing FLAG-Nectin-4 were serumstarved and treated with the ErbB2 inhibitor irbinitinib at $1 \mu \mathrm{M}$, the PI3K inhibitor wortmannin at $1 \mu \mathrm{M}$ or the PI3K inhibitor LY294002 at $50 \mu \mathrm{M}$, the MEK inhibitor U0126 at $10 \mu \mathrm{M}$, or the JAK1 and JAK2 inhibitor ruxolitinib at $1 \mu \mathrm{M}$. The assay was carried out as in $(\mathbf{a}-\mathbf{c})$. Bars indicate the means \pm S.E. of three independent experiments. The actual $P$ values for each test are shown in each figure. Scale bars, $100 \mu \mathrm{m}$. Representative results (images) from three independent experiments are shown.

or rec-FLAG-Nectin-4-Ig3 was incubated with the ErbB2-GFP-immobilized beads, both rec-FLAG-Nectin4-Ig1/2/3 and rec-FLAG-Nectin-4-Ig3 were co-immunoprecipitated with ErbB2-GFP (Supplementary Fig. 3a,b,e). When both recombinant proteins were simultaneously incubated with ErbB2-GFP, the amount of rec-FLAG-Nectin-4-Ig1/2/3 co-precipitated with ErbB2-GFP was reduced in the presence of rec-FLAG-Nectin4-Ig3, and vice versa (Supplementary Fig. 3c,e). This result indicates that the third Ig-like domain of nectin-4 is required for the cis-interaction of full-length nectin-4 with ErbB2. Together with the above result showing that the third Ig-like domain of nectin-4 alone interacts with ErbB2, these results indicate that the third Ig-like domain of nectin- 4 is required and sufficient for its cis-interaction with ErbB2.

Enhancement of the homodimerization and tyrosine-phosphorylation of ErbB2, activation of AKT, and DNA synthesis by the third Ig-like domain of nectin-4. We then examined whether the third Ig-like domain of nectin-4 enhances the homodimerization of ErbB2. ErbB2-HA and ErbB2-GFP were co-expressed with FLAG-Nectin-4-Ig3-TM or FLAG as control in HEK293E cells. When ErbB2-GFP was immunoprecipitated with the anti-GFP pAb, ErbB2-HA was co-immunoprecipitated with ErbB2-GFP and FLAG-Nectin-4-Ig3-TM, but not FLAG, and the amount of ErbB2-HA from the cells co-expressing 
a

IB:

HA

GFP

FLAG

ErbB2-HA

ErbB2-GFP

FLAG-Nectin-4-Ig3-TM

C Control
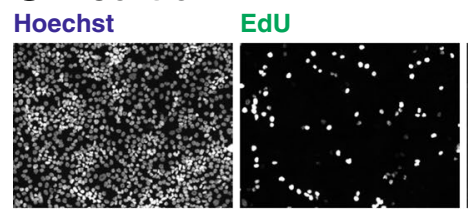

FLAG

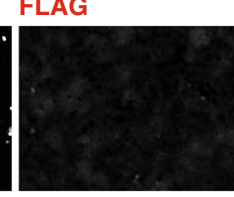

Merge
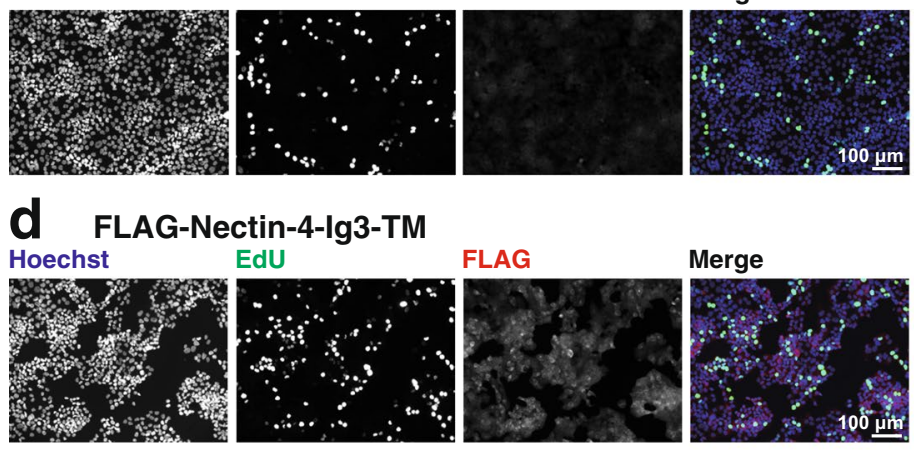

e

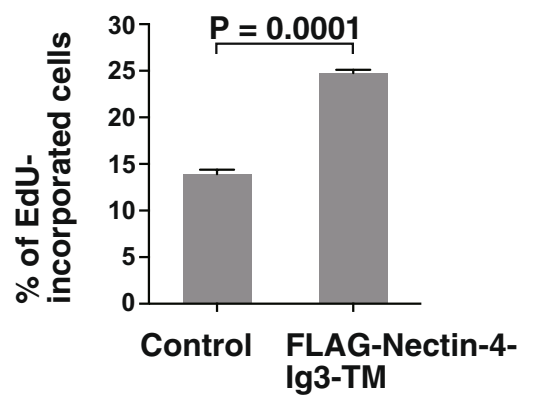

b

IB:

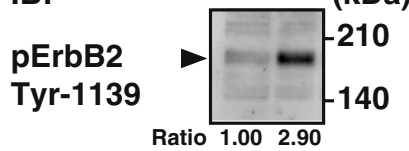

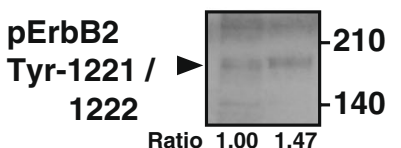

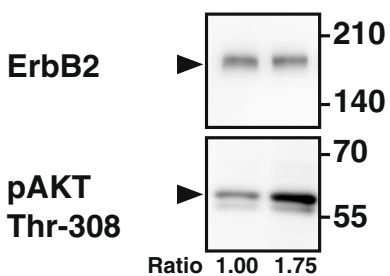

AKT
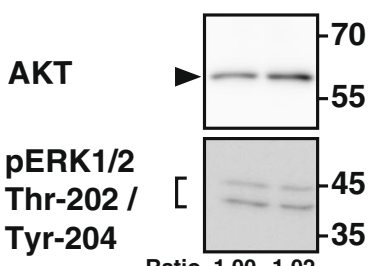

Tyr-204

Ratio 1.001 .02
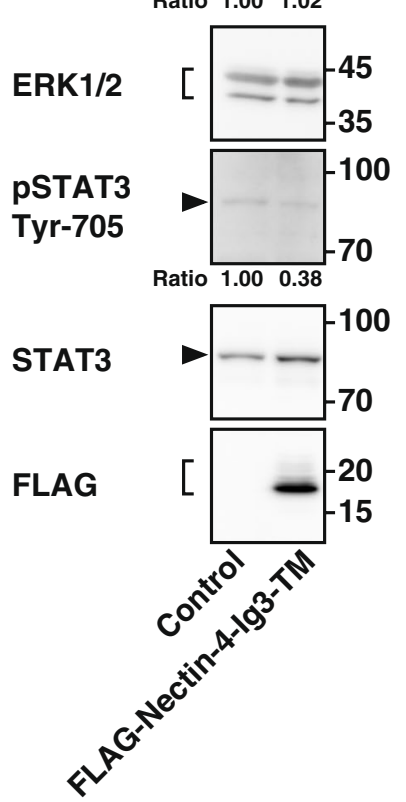

Figure 6. Enhancement of the homodimerization and tyrosine-phosphorylation of ErbB2, AKT activation, and DNA synthesis by the third immunoglobulin-like domain of nectin-4. (a) Enhancement of the homodimerization of ErbB2 by the third immunoglobulin (Ig)-like domain of nectin-4. HEK293E cells were co-transfected with various combinations of the indicated plasmids and cultured in suspension. GFP-tagged ErbB2 (ErbB2-GFP) was immunoprecipitated using the anti-GFP pAb. The samples were subjected to Western blotting using the indicated Abs. Ratio represents the band intensities of the co-immunoprecipitated HA-tagged ErbB2 (ErbB2-HA) that were normalized to those of the immunoprecipitated ErbB2-GFP, and the normalized value of the both ErbB2-HA and ErbB2-GFP, but not FLAG-tagged third Ig-like domain of nectin-4 (FLAGNectin-4-Ig3-TM), expressing cells was set as 1.00. (b) Enhancement of the AKT activation by the third Ig-like domain of nectin-4. T47D cells stably expressing FLAG-Nectin-4-Ig3-TM were serum-starved for $24 \mathrm{~h}$, and the samples were subjected to Western blotting using the indicated Abs. Ratio represents the band intensities of each phosphorylated protein on the indicated tyrosine and threonine residues that were normalized to those of each total protein, and the normalized value of the control cells was set as 1.00. Arrowheads and square brackets indicate each of the proteins. The displayed blots were cropped, and the full-length blots are shown in Supplementary Fig. 8. Representative results from three independent experiments are shown. (c-e) T47D control cells (c) or T47D cells stably expressing FLAG-Nectin-4-Ig3-TM (d) were serum-starved and treated with medium containing $10 \mu \mathrm{M}$ EdU for $12 \mathrm{~h}$. After washout of the medium, the cells were cultured in 
the absence of serum and stained with the anti-DDDDK mAb, Hoechst33342, and EdU staining reagents. The number of EdU-incorporated cells was counted by microscopy and Hybrid Cell Counter software (e). Bars indicate the means \pm S.E. of three independent experiments. The actual $P$ values for each test are shown in each figure. Scale bars, $100 \mu \mathrm{m}$ IB, immunoblotting; IP, immunoprecipitation. Representative results (images) from three independent experiments are shown.

FLAG-Nectin-4-Ig3-TM was more than that co-immunoprecipitated with ErbB2-GFP from the control cells (Fig. 6a). These results indicate that the cis-interaction of the third Ig-like domain of nectin-4 with ErbB2 enhances its homodimerization.

We further examined whether the third Ig-like domain of nectin-4 enhances the tyrosine-phosphorylation of ErbB2, activation of AKT, and DNA synthesis. For this purpose, we established a cell line stably expressing FLAG-Nectin-4-Ig3-TM in T47D cells. The tyrosine-phosphorylation of ErbB2 and the threonine-phosphorylation of AKT were enhanced in the T47D cells stably expressing FLAG-Nectin-4-Ig3-TM compared with those in the control cells, whereas the threonine- and tyrosine-phosphorylation of ERK1/2 or the tyrosine-phosphorylation of STAT3 was not significantly enhanced in the T47D cells stably expressing FLAG-Nectin-4-Ig3-TM compared with those in the control cells (Fig. 6b). The incorporation of EdU into the T47D cells stably expressing FLAG-Nectin-4-Ig3-TM was enhanced compared with that in the control cells (Fig. 6c-e). These results collectively indicate that the cis-interaction of the third Ig-like domain of nectin- 4 with ErbB2 enhances the homodimerization, leading to the activation of ErbB2 and AKT and DNA synthesis, and that the cytoplasmic region of nectin- 4 is not required for these reactions.

Cis-interaction of nectin-4 with trastuzumab-resistant variants of ErbB2 and enhancement of their signalling pathways for DNA synthesis. The ErbB2 splice variants, p95-ErbB2 and ErbB2 $\Delta$ Ex16, were shown to be trastuzumab-resistant in breast cancers ${ }^{13,15,17}$, although the trastuzumab-resistance to ErbB2 $\Delta$ Ex16 is controversial ${ }^{21,22}$. The schematic diagrams and amino acid sequences of these variants are shown in Fig. 7a. We examined whether nectin-4 cis-interacts with these variants. FLAG-Nectin-4 was co-expressed with p95-ErbB2-GFP or ErbB2 $\triangle$ Ex16-GFP in HEK293E cells. The cells were cultured in suspension. When FLAG-Nectin-4 was immunoprecipitated using the anti-FLAG mAb, both p95-ErbB2-GFP and ErbB2 $\Delta$ Ex16-GFP were co-immunoprecipitated with it to a similar extent (Fig. 7b). These results indicate that nectin- 4 cis-interacts with p95-ErbB2 and ErbB2 $\Delta$ Ex16 as well as ErbB2.

We next examined whether the cis-interaction of nectin- 4 with p95-ErbB2 and ErbB2 $\Delta$ Ex16 enhances their tyrosine phosphorylation and AKT activation. For this purpose, we established cell lines stably co-expressing FLAG-Nectin-4 and either p95-ErbB2-GFP or ErbB2 $\triangle$ Ex16-GFP in T47D cells. As a control, we also established cell lines stably expressing either of these variants in T47D cells. The phosphorylation of the tyrosine residues of p95-ErbB2-GFP that are equivalent to tyrosine residues at 1139, 1221, and 1222 of wild-type ErbB2, and the threonine-phosphorylation of AKT and the tyrosine-phosphorylation of STAT3 were enhanced in the T47D cells stably expressing FLAG-Nectin-4 and p95-ErbB2-GFP compared with that in the control cells, whereas the threonine- and tyrosine-phosphorylation of ERK1/2 was not significantly enhanced in the T47D cells stably expressing FLAG-Nectin-4 and p95-ErbB2-GFP compared with that in the control cells (Fig. 7c). The threonine-phosphorylation of AKT, but not the phosphorylation of tyrosine residues of ErbB2 $\Delta$ Ex16-GFP that are equivalent to tyrosine residues at 1139, 1221, and 1222 of wild-type ErbB2, the threonine- and tyrosine-phosphorylation of ERK1/2, or the tyrosine-phosphorylation of STAT3, was enhanced in the T47D cells stably expressing FLAG-Nectin-4 and ErbB2 $\triangle$ Ex16-GFP compared with those in the control cells (Fig. 7c). The incorporation of EdU into the T47D cells stably co-expressing FLAG-Nectin-4 and either p95-ErbB2-GFP or ErbB2 $\Delta$ Ex16-GFP was enhanced compared with that in the control cells (Fig. 7d). The incorporation of EdU into DNA in the T47D cells stably expressing FLAG-Nectin-4 and p95-ErbB2-GFP or ErbB2 $\triangle$ Ex16-GFP and the control cells expressing p95-ErbB2-GFP or ErbB2 $\Delta$ Ex16-GFP alone was inhibited by wortmannin, LY294002, and U0126, but not by ruxolitinib (Fig. 7e). These results collectively indicate that nectin-4 cis-interacts with not only ErbB2 but also p95-ErbB2 and ErbB2 $\Delta$ Ex16, that nectin- 4 enhances the p95-ErbB2- and ErbB2 $\Delta$ Ex16-mediated DNA synthesis through the activation of the PI3K-AKT signalling pathway, and that the nectin-4-independent p95-ErbB- and ErbB $\Delta$ Ex16-mediated activation of the Ras-Raf-MEK-ERK1/2 signalling pathway, but not the JAK-STAT3 signalling pathway, is also involved in the p95-ErbB- and ErbB $\Delta$ Ex16-mediated DNA synthesis.

Schematic diagrams for the modes of action of nectin-4 on the signalling pathways of ErbB2 and its trastuzumab-resistant variants for DNA synthesis. The modes of action of nectin- 4 on the signalling pathways of ErbB2 and its trastuzumab-resistant variants for DNA synthesis are schematically demonstrated in Fig. 8a-c. As shown in Fig. 8a, nectin-4 cis-interacted with ErbB2 and enhanced its homodimerization and tyrosine-phosphorylation. This interaction selectively induced the activation of the PI3K-AKT signalling pathway for DNA synthesis, but not the Ras-Raf-MEK-ERK1/2 or JAK-STAT3 signalling pathway. As shown in Fig. 8b,c, nectin-4 cis-interacted with not only ErbB2 but also its trastuzumab-resistant splice variants, p95-ErbB2 and ErbB2 $\Delta$ Ex16, that are homodimerized through the disulfide bond of two cysteine residues ${ }^{10,11,16,17}$. The cis-interaction of nectin-4 with p95-ErbB2 enhanced the phosphorylation of its tyrosine residues at 1139, 1221, and 1222 as well as the threonine-phosphorylation of AKT, similar to that with ErbB2, but additionally enhanced the tyrosine-phosphorylation of STAT3 that was not augmented by the cis-interaction of nectin- 4 with ErbB2 or ErbB2 $\Delta$ Ex16, as shown in Fig. $8 \mathrm{a}-\mathrm{c}$. Although the $c i$ s-interaction of nectin- 4 with ErbB2 $\Delta$ Ex16 did not enhance the phosphorylation of its tyrosine residues at 1139, 1221, or 1222, the threonine-phosphorylation of AKT was enhanced similar to that with ErbB2 as shown in Fig. 8c. These models may provide a clue about a mechanism 


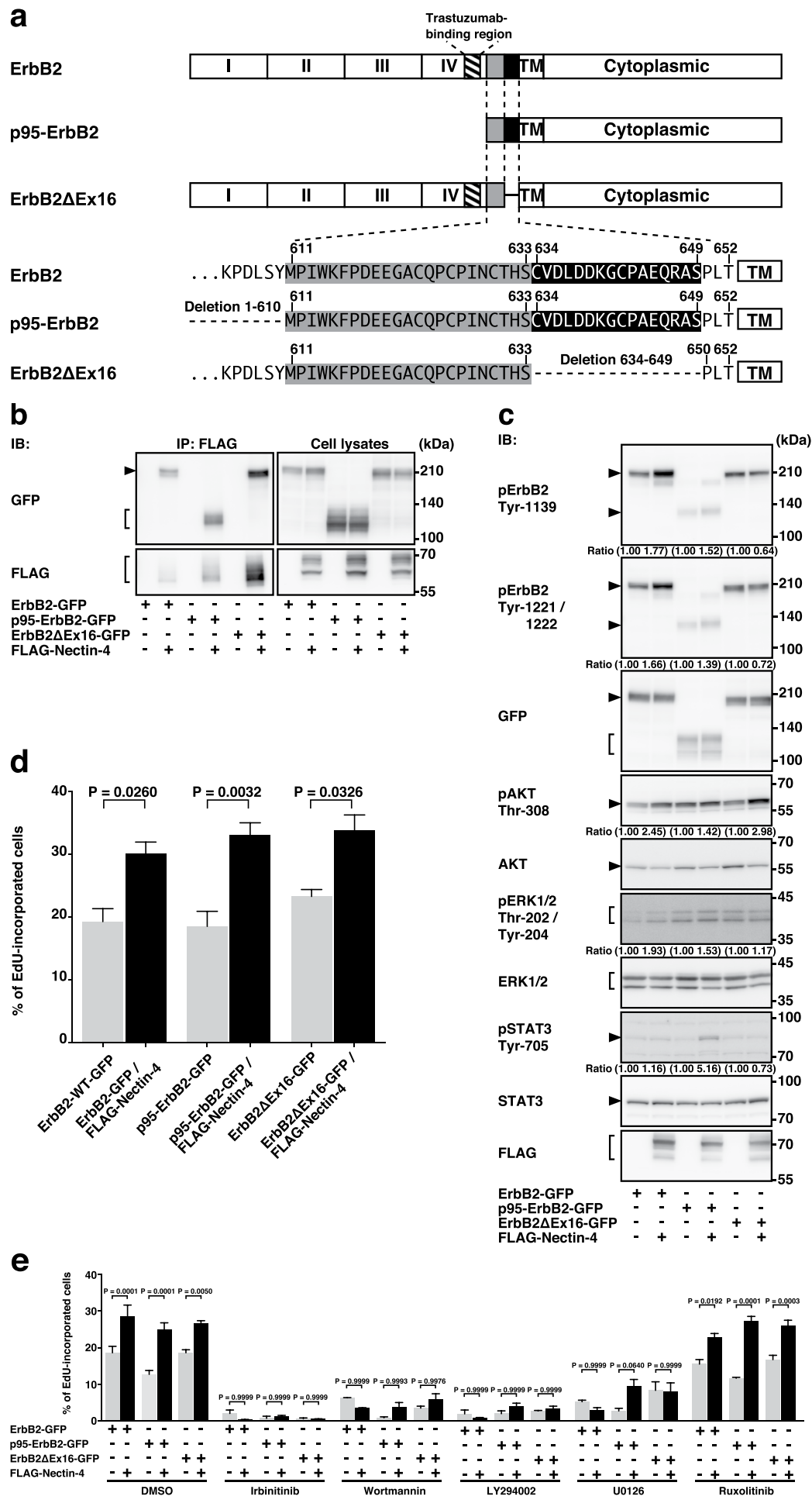

Figure 7. Cis-interaction of nectin-4 with trastuzumab-resistant variants of ErbB2 and enhancement of their signalling pathways for DNA synthesis. (a) Schematic diagram and amino acid sequences of ErbB2 splice variants. (b) Cis-interaction of nectin-4 with ErbB2 splice variants. HEK293E cells were co-transfected with various combinations of the indicated plasmids and cultured in suspension. FLAG-tagged nectin-4 (FLAGNectin-4) was immunoprecipitated using the anti-FLAG mAb. The samples were subjected to Western blotting using the indicated Abs. (c) Enhancement of the tyrosine-phosphorylation of ErbB2 splice variants and the PI3K-AKT signalling pathway by nectin-4. T47D cells stably expressing ErbB2 splice variants or T47D cells stably expressing GFP-tagged ErbB2 splice variants with FLAG-Nectin-4 were serum-starved for $24 \mathrm{~h}$, and the samples were subjected to Western blotting using the indicated Abs. Ratio represents the band intensities of each phosphorylated protein on the indicated tyrosine and threonine residues that were normalized to those of each total protein, and the normalized value of each control cell (GFP-tagged ErbB2 and its splice variants, but not FLAG-Nectin-4, expressing cells) was set as 1.00. Arrowheads and square brackets indicate each of 
the proteins. The displayed blots were cropped, and the full-length blots are shown in Supplementary Fig. 9. Representative results from three independent experiments are shown. (d) Enhancement of DNA synthesis by nectin-4 in ErbB2 splice variant-expressing cells. T47D cells stably expressing ErbB2 splice variants or T47D cells stably expressing GFP-tagged ErbB2 splice variants with FLAG-Nectin-4 were serum-starved and treated with medium containing $10 \mu \mathrm{M}$ EdU for $12 \mathrm{~h}$. After washout of the medium, the cells were cultured in the absence of serum and stained with Hoechst33342, and EdU staining reagents. The number of EdU-incorporated cells was counted by microscopy and Hybrid Cell Counter software. (e) Reduction of DNA synthesis by the ErbB2, PI3K, and MEK inhibitors, but not the JAK inhibitor in ErbB2 splice variant-expressing cells. T47D cells stably expressing ErbB2 splice variants or T47D cells stably expressing GFP-tagged ErbB2 splice variants with FLAG-Nectin- 4 were serum-starved and treated with the ErbB2 inhibitor irbinitinib at $1 \mu \mathrm{M}$, the PI3K inhibitor wortmannin at $1 \mu \mathrm{M}$ or the PI3K inhibitor LY294002 at $50 \mu \mathrm{M}$, the MEK inhibitor U0126 at $10 \mu \mathrm{M}$, or the JAK1 and JAK2 inhibitor ruxolitinib at $1 \mu \mathrm{M}$. The assay was carried out as in (d). Bars indicate the means \pm S.E. of three independent experiments. The actual $\mathrm{P}$ values for each test are shown in each figure. pAKT, phosphoAKT; pErbB2, phospho-ErbB2; pERK1/2, phospho-ERK1/2; pSTAT3, phospho-STAT3; IB, immunoblotting; IP, immunoprecipitation.

how the activities of oncogenic cell surface receptors, such as ErbB2 and trastuzumab-resistant splice variants, p95-ErbB2 and ErbB2 $\Delta$ Ex16, are regulated by nectin- 4 as a co-receptor.

\section{Discussion}

We showed here for the first time that nectin-4 cis-interacted with ErbB2 through the third Ig-like domain of nectin-4 and domain IV of ErbB2 and enhanced its homodimerization and tyrosine-phosphorylation, leading to the selective activation of the PI3K-AKT signalling pathway for DNA synthesis, but not the Ras-Raf-MEK-ERK1/2 or JAK-STAT3 signalling pathway. It was previously shown that the homodimerization of ErbB2 requires its upregulation, although the ligand-dependent homodimerization of ErbB1 or the heterodimerization of ErbB2 with ErbB3 does not require the upregulation of these molecules ${ }^{1-5}$, indicating that the affinity for the interaction between the extended domain II of one ErbB2 molecule and that of another ErbB2 molecule might be lower than the affinity of the interaction between the extended domain II of ErbB1 and that of another ErbB1 molecule as well as the affinity of the interaction between the extended domain II of one ErbB2 molecule and that of another ErbB3 molecule. The cis-interaction of nectin-4 with ErbB2 is likely to increase the affinity for the interaction between the extended domain II of one ErbB2 molecule and that of another ErbB2 molecule.

We further showed here that the knockdown of endogenous nectin-4 reduced the tyrosine-phosphorylation of ErbB2 and its activation. However, the level of the tyrosine-phosphorylation of ErbB2 was not always correlated with the expression levels of nectin-4. The exact reason for this discrepancy is not clear, but it was previously shown that the growth factor receptors could dimerize depending on their expression levels and that this dimerization of the receptors enhances their tyrosine-phosphorylation and the activation of their downstream signalling pathways $^{1-5}$. Taken together these previous observations, the present results suggest that nectin- 4 is an important co-receptor regulating the tyrosine-phosphorylation level of ErbB2 but may not be the only molecule showing this activity.

It was noted that the cis-interaction of nectin- 4 with ErbB2 induces the activation of the PI3K-AKT signalling pathway more efficiently than that of the Ras-Raf-MEK-ERK1/2 and JAK-STAT3 signalling pathways. It was previously shown that the Ras-Raf-MEK-ERK signalling pathway is a major mitogenic signalling pathway of ErbB2, but it was subsequently demonstrated that the PI3K-AKT signalling pathway is also a major mitogenic signalling pathway of ErbB2 ${ }^{62,63}$. It was more recently reported that upregulated ErbB2 enhances the phosphorylation of tyrosine residues at $1139,1221,1222$, and 1248 of ErbB2 ${ }^{61}$. The mutation of tyrosine residue at 1139 reduces the phosphorylation of AKT, indicating that the tyrosine phosphorylation of this residue on ErbB2 at least leads to the activation of the PI3K-AKT signalling pathway ${ }^{61}$. It remains unclear whether the phosphorylation of other tyrosine residues leads to the activation of the PI3K-AKT signalling pathway. The novel stimulatory mechanism of ErbB2 for its homodimerization and activation by cis-interacting with nectin-4, which mainly leads to the selective activation of the PI3K-AKT signalling pathway, may be involved in the enhanced DNA synthesis of cancer cells in which nectin- 4 is upregulated. The novel mechanism presented here was consistent with the previous observations that the PI3K-AKT signalling pathway is implicated in the carcinogenic role of nectin-4 in gallbladder, breast, and gastric cancers ${ }^{50,51,53}$.

We further demonstrated here that nectin-4 cis-interacted with the trastuzumab-resistant splice variants of ErbB2, p95-ErbB2 and ErbB2 $\Delta$ Ex16, although the trastuzumab-resistance to ErbB2 $\Delta$ Ex16 is controversial $^{21,22}$. It was previously shown that trastuzumab interacts with domain IV of ErbB2, but p95-ErbB2 lacks the trastuzumab-interacting region, which is considered as a mechanism for trastuzumab resistance of p95-ErbB2-expressing breast cancers ${ }^{13,15,17}$. In contrast, the trastuzumab-interacting region is intact in ErbB2 $\Delta$ Ex16, but the efficiency of trastuzumab for its interaction with this region of ErbB2 $\Delta$ Ex16 is less than that of ErbB2 ${ }^{11}$. Considering from our data that nectin- 4 cis-interacted with p95-ErbB2 and ErbB2 $\Delta$ Ex16, the nectin-4-interacting region in domain IV of ErbB2 is likely different from the trastuzumab-interacting region.

We finally showed here that the cis-interaction of nectin-4 with p95-ErbB2 enhanced the phosphorylation of its tyrosine residues at 1139, 1221, and 1222 as well as the threonine-phosphorylation of AKT and additionally enhanced the tyrosine-phosphorylation of STAT3. The mechanism for this action of p95-ErbB2 remains unknown, but the phosphorylation of tyrosine residues other than 1139, 1221, and 1222 may be enhanced by this interaction. It was noted that the cis-interaction of nectin- 4 with ErbB2 $\Delta$ Ex 16 enhanced the tyrosine-phosphorylation of AKT, but not the phosphorylation of tyrosine residues at 1139, 1221, and 1222 of 
a

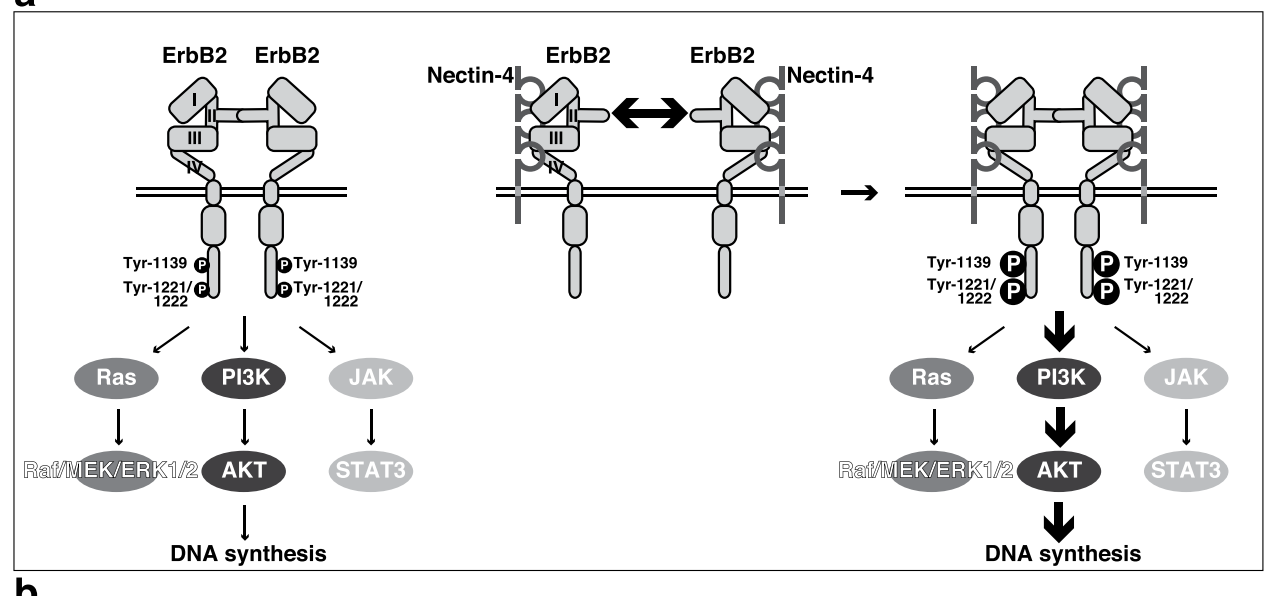

b

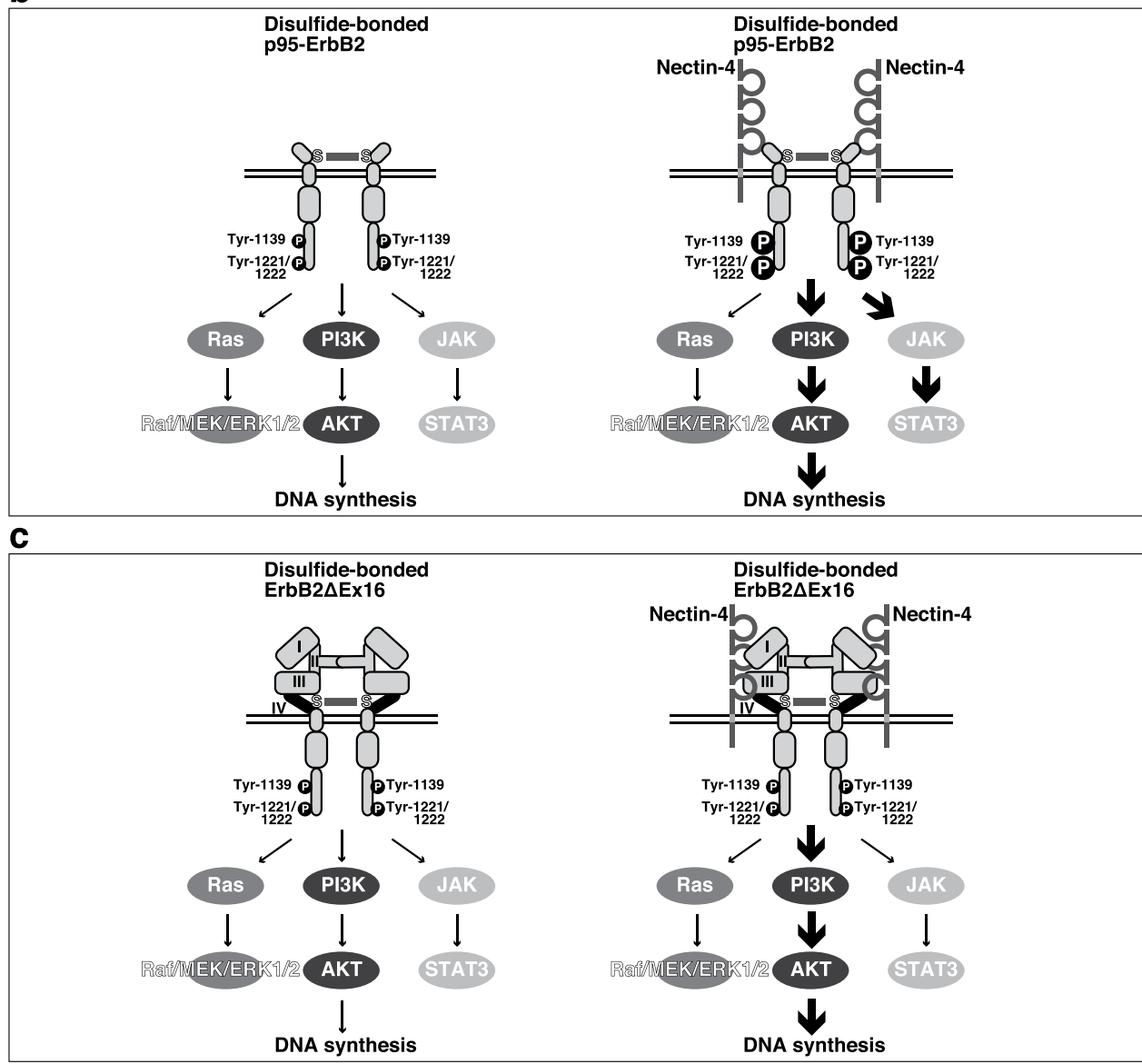

Figure 8. Schematic diagrams for the modes of action of nectin-4 on the signalling pathways of ErbB2 and its trastuzumab-resistant variants for DNA synthesis. (a) A mechanism for ErbB2. (b) A mechanism for p95ErbB2. (c) A mechanism for ErbB2 $\Delta$ Ex16.

ErbB2 $\Delta$ Ex16. The phosphorylation of tyrosine residues other than these residues may be enhanced by this interaction. Thus, nectin-4 cis-interacts with ErbB2, p95-ErbB2, and ErbB2 $\Delta$ Ex16, but this interaction may enhance the phosphorylation of different tyrosine residues and/or another unidentified molecule. In contrast to ErbB2, p95-ErbB2 and ErbB2 $\triangle$ Ex16 are homodimerized through the disulfide bond of two cysteine residues ${ }^{10,11,16,17}$. Therefore, it is likely that nectin-4 cis-interacts with the dimerized molecule, enhancing their activation, although nectin- 4 cis-interacts with the monomer of ErbB2 to form a complex, enhancing its dimerization. The molecular mechanisms for the nectin-4-enhanced activation of p95-ErbB2 and ErbB2 $\Delta$ Ex16 remain unknown, but the binding of nectin- 4 to domain IV of these molecules may affect the conformational changes of their cytoplasmic regions so that the intermolecular tyrosine-phosphorylation is enhanced.

Enfortumab vedotin, an Ab-drug conjugate targeting nectin-4, has been developed as a highly potent therapeutic agent in multiple preclinical cancer model ${ }^{58}$. This $\mathrm{Ab}$ recognizes the first Ig-like domain of nectin-4 
and inhibits its trans-interaction with nectin-1. Targeting the third Ig-like domain of nectin-4, which cis-interacts with ErbB2, might be an alternative strategy for the treatment of trastuzumab-resistant breast cancers expressing p95-ErbB2 and ErbB2 $\Delta$ Ex16, although it was recently reported that trastuzumab is effective for breast cancers expressing ErbB2 $\Delta \mathrm{Ex} 16^{21,22}$. This novel strategy for the development of therapeutic drugs for trastuzumab-resistant breast cancers may be also effectively used for the screening of chemical compound drugs.

\section{Methods}

Cell culture and transfection. The method of cell culture was described in Supplementary Methods. Transfection reagents and supplements for cells used in this study were listed in Supplementary Table 1.

Plasmid construction. The cDNAs used in this study were described in Supplementary Methods.

Antibodies and reagents. The Abs and reagents used in this study were listed in Supplementary Table 2 and Supplementary Table 1, respectively. Rabbit anti-Necl-1 pAb, rabbit anti-Necl-2 pAb, and rabbit anti-Necl-3 pAb were described previously ${ }^{64-66}$.

Co-immunoprecipitation assay and Western blotting. The methods of co-immunoprecipitation assay and Western blotting were described in Supplementary Methods. The reagents for the assays were listed in Supplementary Table 1.

Immunofluorescence microscopy. The methods of confocal image analysis were described in Supplementary Methods. The Abs for the assays were listed in Supplementary Table 2.

Assay for the phosphorylation of ErbB2, AKT, ERK1/2, and STAT3. T47D or SUM190-PT cells were plated at a density of $3 \times 10^{4}$ cells per square centimetre on dishes and cultured for 2 days. The cells were serum-starved with RPMI-1640 or Ham's F12 containing $0.5 \%$ fatty acid-free BSA in the absence or presence of the inhibitor at $37^{\circ} \mathrm{C}$ for $24 \mathrm{~h}$, and then the cells were washed with ice-cold PBS and lysed with the lysis buffer. The lysates were then heated at $80^{\circ} \mathrm{C}$ in the SDS sample buffer for $2 \mathrm{~min}$ and subjected to SDS-PAGE, followed by Western blotting. The intensity of the bands in Western blotting was calculated using ImageJ $1.48 \mathrm{v} 32$-bit software.

siRNA experiments. Silencer select siRNA against human NECTIN4 and a silencer select siRNA negative control siRNA (AM4611) were purchased (Thermo Fisher Scientific, Waltham, MA, USA). Transfection reagent and siRNA sequences were listed in Supplementary Tables 1 and 3, respectively.

Assay for DNA synthesis. T47D or SUM190-PT cells were plated at a density of $3 \times 10^{4}$ cells per square centimetre on dishes and cultured at $37^{\circ} \mathrm{C}$ for $48 \mathrm{~h}$. The cells were starved of serum, cultured with RPMI1640 or Ham's F12 containing $0.5 \%$ fatty acid-free BSA at $37^{\circ} \mathrm{C}$ in the absence or presence of the inhibitors for $24 \mathrm{~h}$ and then treated with $10 \mu \mathrm{M} \mathrm{EdU}$ at $37^{\circ} \mathrm{C}$ for $12 \mathrm{~h}$. After washout of EdU-containing media, EdU-treated cells were additionally cultured with serum-free medium in the absence or presence of the inhibitors at $37^{\circ} \mathrm{C}$ for $12 \mathrm{~h}$ and fixed using 4\% paraformaldehyde diluted in PBS. The signal for EdU and nuclei in the cells was determined by the Click-iT EdU Alexa Fluor 488 or 647 Imaging Kit (Thermo Fisher Scientific), according to the manufacturer's protocol. The samples were counted by fluorescence microscopic examination. The images were acquired using a BZ-X710 microscope (KEYENCE CORPORATION, Osaka, Japan) with a CFI Plan Apo $\lambda 20 \times / 0.75$ numerical aperture objective lens (Nikon, Inc., Tokyo, Japan) in $1,920 \times 1,440$ pixels. The displayed images were applied into maximum signal intensity projection from around 5 confocal images collected at a $2 \mu \mathrm{m}$ step along the $\mathrm{z}$-axis at room temperature under the control of BZ-X Viewer software (KEYENCE CORPORATION). The images were processed using ImageJ $1.48 \mathrm{v} 32$-bit software.

Protein purification and in vitro assay for the inhibition of the interaction between nectin- 4 and ErbB2. The method of the purification of recombinant proteins was described in Supplementary Methods. Inhibition of the interaction between the extracellular region of nectin-4 and ErbB2 was carried out using recombinant FLAG-tagged nectin-4 Ig1/2/3 and ErbB2-GFP by adding recombinant FLAG-tagged nectin-4 Ig3. ErbB2-GFP was captured on anti-GFP pAb-covalently-conjugated beads by incubating with the lysates of HEK293E cells expressing ErbB2-GFP, and the beads were washed three times with the lysis buffer. The washed beads were resuspended in the lysis buffer containing $5 \mu \mathrm{M}$ recombinant FLAG-tagged nectin- 4 Ig1/2/3 in the presence or absence of $25 \mu \mathrm{M}$ recombinant FLAG-tagged nectin-4 Ig3 and incubated in a total volume of $20 \mu \mathrm{l}$ at $4^{\circ} \mathrm{C}$ for $16 \mathrm{~h}$ with gentle mixing. After ErbB2-GFP were mixed with the recombinant proteins, the beads were washed three times with PBS containing $0.02 \%$ Tween-20, and the bound proteins were eluted by heating at $80^{\circ} \mathrm{C}$ in the SDS sample buffer for $2 \mathrm{~min}$. The samples were subjected to SDS-PAGE, followed by Western blotting using the indicated Abs.

Statistical analysis. Statistical significance was analysed using GraphPad Prism 6 software (GraphPad Software Inc., La Jolla, CA, USA) for two-tailed Welch's t-test for two groups, or one-way analysis of variance followed by a post hoc Tukey's test for comparisons among more than three groups.

\section{Data availability}

All of the datasets analyzed and all of the reagents used or generated during this study are available from the corresponding authors on reasonable request. 
Received: 13 August 2019; Accepted: 26 November 2019; Published online: 12 December 2019

\section{References}

1. Yarden, Y. \& Sliwkowski, M. X. Untangling the ErbB signalling network. Nat Rev Mol Cell Biol 2, 127-137 (2001)

2. Citri, A. \& Yarden, Y. EGF-ERBB signalling: towards the systems level. Nat Rev Mol Cell Biol 7, 505-516 (2006).

3. Lemmon, M. A. \& Schlessinger, J. Cell signaling by receptor tyrosine kinases. Cell 141, 1117-1134 (2010).

4. Lemmon, M. A., Schlessinger, J. \& Ferguson, K. M. The EGFR family: not so prototypical receptor tyrosine kinases. Cold Spring Harb Perspect Biol 6, a020768 (2014).

5. Roskoski, R. Jr. The ErbB/HER family of protein-tyrosine kinases and cancer. Pharmacol Res 79, 34-74 (2014).

6. Slamon, D. J. et al. Human breast cancer: correlation of relapse and survival with amplification of the HER-2/neu oncogene. Science 235, 177-182 (1987).

7. Slamon, D. J. et al. Studies of the HER-2/neu proto-oncogene in human breast and ovarian cancer. Science 244, 707-712 (1989).

8. Swain, S. M. et al. Pertuzumab, trastuzumab, and docetaxel in HER2-positive metastatic breast cancer. N Engl J Med 372, 724-734 (2015).

9. Kwong, K. Y. \& Hung, M. C. A novel splice variant of HER2 with increased transformation activity. Mol Carcinog 23, 62-68 (1998).

10. Siegel, P. M., Ryan, E. D., Cardiff, R. D. \& Muller, W. J. Elevated expression of activated forms of Neu/ErbB-2 and ErbB-3 are involved in the induction of mammary tumors in transgenic mice: implications for human breast cancer. EMBO J 18, 2149-2164 (1999).

11. Castiglioni, F. et al. Role of exon-16-deleted HER2 in breast carcinomas. Endocr Relat Cancer 13, 221-232 (2006).

12. Anido, J. et al. Biosynthesis of tumorigenic HER2 C-terminal fragments by alternative initiation of translation. EMBO J 25, 3234-3244 (2006).

13. Scaltriti, M. et al. Expression of p95HER2, a truncated form of the HER2 receptor, and response to anti-HER2 therapies in breast cancer. J Natl Cancer Inst 99, 628-638 (2007).

14. Mitra, D. et al. An oncogenic isoform of HER2 associated with locally disseminated breast cancer and trastuzumab resistance. Mol Cancer Ther 8, 2152-2162 (2009).

15. Scaltriti, M. et al. Clinical benefit of lapatinib-based therapy in patients with human epidermal growth factor receptor 2-positive breast tumors coexpressing the truncated p95HER2 receptor. Clin Cancer Res 16, 2688-2695 (2010).

16. Marchini, C. et al. The human splice variant $\triangle 16$ HER2 induces rapid tumor onset in a reporter transgenic mouse. PLoS One 6, e18727 (2011).

17. Arribas, J., Baselga, J., Pedersen, K. \& Parra-Palau, J. L. p95HER2 and breast cancer. Cancer Res 71, 1515-1519 (2011).

18. Nahta, R. Molecular mechanisms of trastuzumab-based treatment in HER2-overexpressing breast cancer. ISRN Oncol 2012, 428062 (2012).

19. Turpin, J. et al. The ErbB2 $\Delta$ Ex16 splice variant is a major oncogenic driver in breast cancer that promotes a pro-metastatic tumor microenvironment. Oncogene 35, 6053-6064 (2016).

20. Nami, B. \& Wang, Z. HER2 in breast cancer stemness: A negative feedback loop towards trastuzumab resistance. Cancers (Basel) 9 (2017).

21. Alajati, A. et al. Mammary tumor formation and metastasis evoked by a HER2 splice variant. Cancer Res 73, 5320-5327 (2013).

22. Castagnoli, L. et al. Activated d16HER2 homodimers and SRC kinase mediate optimal efficacy for trastuzumab. Cancer Res 74, 6248-6259 (2014).

23. Molina, M. A. et al. $\mathrm{NH}_{2}$-terminal truncated HER-2 protein but not full-length receptor is associated with nodal metastasis in human breast cancer. Clin Cancer Res 8, 347-353 (2002).

24. Sáez, R. et al. p95HER-2 predicts worse outcome in patients with HER-2-positive breast cancer. Clin Cancer Res 12, 424-431 (2006).

25. Ferguson, K. M. Structure-based view of epidermal growth factor receptor regulation. Annu Rev Biophys 37, 353-373 (2008).

26. Kaplan, M. et al. EGFR dynamics change during activation in native membranes as revealed by NMR. Cell 167, 1241-1251 e1211 (2016).

27. Moasser, M. M. The oncogene HER2: its signaling and transforming functions and its role in human cancer pathogenesis. Oncogene 26, 6469-6487 (2007).

28. Ursini-Siegel, J., Schade, B., Cardiff, R. D. \& Muller, W. J. Insights from transgenic mouse models of ERBB2-induced breast cancer. Nat Rev Cancer 7, 389-397 (2007)

29. Wagner, M. J., Stacey, M. M., Liu, B. A. \& Pawson, T. Molecular mechanisms of SH2- and PTB-domain-containing proteins in receptor tyrosine kinase signaling. Cold Spring Harb Perspect Biol 5, a008987 (2013).

30. Shah, D. \& Osipo, C. Cancer stem cells and HER2 positive breast cancer: The story so far. Genes Dis 3, 114-123 (2016).

31. Mishra, R., Hanker, A. B. \& Garrett, J. T. Genomic alterations of ERBB receptors in cancer: clinical implications. Oncotarget 8, 114371-114392 (2017)

32. Shang, A. Q. et al. Relationship between HER2 and JAK/STAT-SOCS3 signaling pathway and clinicopathological features and prognosis of ovarian cancer. Cancer Biol Ther 18, 314-322 (2017).

33. Yamazaki, H. et al. Amplification of the structurally and functionally altered epidermal growth factor receptor gene $(c$-erbB) in human brain tumors. Mol Cell Biol 8, 1816-1820 (1988).

34. Batra, S. K. et al. Epidermal growth factor ligand-independent, unregulated, cell-transforming potential of a naturally occurring human mutant EGFRvIII gene. Cell Growth Differ 6, 1251-1259 (1995).

35. Cho, H. S. et al. Structure of the extracellular region of HER2 alone and in complex with the Herceptin Fab. Nature 421, 756-760 (2003).

36. Baselga, J. \& Swain, S. M. Novel anticancer targets: revisiting ERBB2 and discovering ERBB3. Nat Rev Cancer 9, 463-475 (2009).

37. Scheuer, W. et al. Strongly enhanced antitumor activity of trastuzumab and pertuzumab combination treatment on HER2-positive human xenograft tumor models. Cancer Res 69, 9330-9336 (2009).

38. Reymond, N. et al. Nectin4/PRR4, a new afadin-associated member of the nectin family that trans-interacts with nectin1/PRR1 through V domain interaction. J Biol Chem 276, 43205-43215 (2001).

39. Takai, Y. \& Nakanishi, H. Nectin and afadin: novel organizers of intercellular junctions. J Cell Sci 116, 17-27 (2003).

40. Takai, Y., Ikeda, W., Ogita, H. \& Rikitake, Y. The immunoglobulin-like cell adhesion molecule nectin and its associated protein afadin. Annu. Rev. Cell Dev. Biol. 24, 309-342 (2008).

41. Takai, Y., Miyoshi, J., Ikeda, W. \& Ogita, H. Nectins and nectin-like molecules: roles in contact inhibition of cell movement and proliferation. Nat Rev Mol Cell Biol 9, 603-615 (2008).

42. Mandai, K., Rikitake, Y., Mori, M. \& Takai, Y. Nectins and nectin-like molecules in development and disease. Curr Top Dev Biol 112, 197-231 (2015).

43. Mizutani, K. \& Takai, Y. Nectin spot: a novel type of nectin-mediated cell adhesion apparatus. Biochem J 473, 2691-2715 (2016).

44. Maruoka, M., Kedashiro, S., Ueda, Y., Mizutani, K. \& Takai, Y. Nectin-4 co-stimulates the prolactin receptor by interacting with SOCS1 and inhibiting its activity on the JAK2-STAT5 a signaling pathway. J Biol Chem 292, 6895-6909 (2017).

45. Mizutani, K., Kedashiro, S., Maruoka, M., Ueda, Y. \& Takai, Y. Nectin-like molecule-4/cell adhesion molecule 4 inhibits the ligandinduced dimerization of ErbB3 with ErbB2. Sci Rep 7, 11375 (2017). 
46. Fabre-Lafay, S. et al. Nectin-4 is a new histological and serological tumor associated marker for breast cancer. BMC Cancer 7, 73 (2007).

47. Takano, A. et al. Identification of nectin-4 oncoprotein as a diagnostic and therapeutic target for lung cancer. Cancer Res 69, 6694-6703 (2009).

48. Derycke, M. S. et al. Nectin 4 overexpression in ovarian cancer tissues and serum: potential role as a serum biomarker. Am J Clin Pathol 134, 835-845 (2010).

49. Nishiwada, S. et al. Nectin-4 expression contributes to tumor proliferation, angiogenesis and patient prognosis in human pancreatic cancer. J Exp Clin Cancer Res 34, 30 (2015).

50. Zhang, Y. et al. A novel PI3K/AKT signaling axis mediates Nectin-4-induced gallbladder cancer cell proliferation, metastasis and tumor growth. Cancer Lett 375, 179-189 (2016).

51. Zhang, Y. et al. Nectin-4 promotes gastric cancer progression via the PI3K/AKT signaling pathway. Hum Pathol 72, 107-116 (2018).

52. Pavlova, N. N. et al. A role for PVRL4-driven cell-cell interactions in tumorigenesis. Elife 2, e00358 (2013).

53. Siddharth, S. et al. Nectin-4 is a breast cancer stem cell marker that induces WNT/ $\beta$-catenin signaling via Pi3k/Akt axis. Int J Biochem Cell Biol 89, 85-94 (2017).

54. Sithanandam, G. \& Anderson, L. M. The ERBB3 receptor in cancer and cancer gene therapy. Cancer Gene Ther 15, 413-448 (2008).

55. Brown, C. W., Amante, J. J. \& Mercurio, A. M. Cell clustering mediated by the adhesion protein PVRL4 is necessary for $\alpha 634$ integrin-promoted ferroptosis resistance in matrix-detached cells. J Biol Chem 293, 12741-12748 (2018).

56. Fabre-Lafay, S. et al. Nectin-4, a new serological breast cancer marker, is a substrate for tumor necrosis factor-alpha-converting enzyme (TACE)/ADAM-17. J Biol Chem 280, 19543-19550 (2005).

57. Msaouel, P., Opyrchal, M., Domingo Musibay, E. \& Galanis, E. Oncolytic measles virus strains as novel anticancer agents. Expert Opin Biol Ther 13, 483-502 (2013).

58. Challita-Eid, P. M. et al. Enfortumab vedotin antibody-drug conjugate targeting nectin-4 is a highly potent therapeutic agent in multiple preclinical cancer models. Cancer Res 76, 3003-3013 (2016).

59. Kwon, Y. K. et al. Activation of ErbB2 during wallerian degeneration of sciatic nerve. J Neurosci 17, 8293-8299 (1997).

60. Muthuswamy, S. K., Gilman, M. \& Brugge, J. S. Controlled dimerization of ErbB receptors provides evidence for differential signaling by homo- and heterodimers. Mol Cell Biol 19, 6845-6857 (1999).

61. Ruiz-Saenz, A. et al. HER2 amplification in tumors activates PI3K/Akt signaling independent of HER3. Cancer Res 78, 3645-3658 (2018).

62. Zhao, D., Sui, Y. \& Zheng, X. MiR-331-3p inhibits proliferation and promotes apoptosis by targeting HER2 through the PI3K/Akt and ERK1/2 pathways in colorectal cancer. Oncol Rep 35, 1075-1082 (2016).

63. Ray, A. Tumor-linked HER2 expression: association with obesity and lipid-related microenvironment. Horm Mol Biol Clin Investig 32 (2017).

64. Kakunaga, S. et al. Nectin-like molecule-1/TSLL1/SynCAM3: a neural tissue-specific immunoglobulin-like cell-cell adhesion molecule localizing at non-junctional contact sites of presynaptic nerve terminals, axons and glia cell processes. J Cell Sci 118, $1267-1277$ (2005).

65. Kawano, S., Ikeda, W., Kishimoto, M., Ogita, H. \& Takai, Y. Silencing of ErbB3/ErbB2 signaling by immunoglobulin-like Necl-2. J Biol Chem 284, 23793-23805 (2009).

66. Yamana, S. et al. The cell adhesion molecule Necl-4/CADM4 serves as a novel regulator for contact inhibition of cell movement and proliferation. PLoS One 10, e0124259 (2015).

\section{Acknowledgements}

We thank Drs. T. Yamamoto (Okinawa Institute of Science and Technology Graduate University, Japan) and S. Higashiyama (Ehime University, Japan) for their generous gifts of reagents. This work was supported by JSPS KAKENHI grant numbers 17K15597 and 17K18142, by MEXT KAKENHI grant number 26114007, by AMED under grant number JP18cm0106111, and a grant from the Japan Foundation for Applied Enzymology. We also thank H. Nikki March, PhD, from Edanz Group (www.edanzediting.com/ac) for editing a draft of this manuscript.

\section{Author contributions}

Y.T. conceived the research project. S.K., K.M. and Y.T. designed the experiments. S.K., A.S. and K.M. performed the experiments. S.K., A.S., K.M. and Y.T. analysed the data and wrote the paper.

\section{Competing interests}

The authors declare no competing interests.

\section{Additional information}

Supplementary information is available for this paper at https://doi.org/10.1038/s41598-019-55460-9.

Correspondence and requests for materials should be addressed to K.M. or Y.T.

Reprints and permissions information is available at www.nature.com/reprints.

Publisher's note Springer Nature remains neutral with regard to jurisdictional claims in published maps and institutional affiliations.

Open Access This article is licensed under a Creative Commons Attribution 4.0 International License, which permits use, sharing, adaptation, distribution and reproduction in any medium or format, as long as you give appropriate credit to the original author(s) and the source, provide a link to the Creative Commons license, and indicate if changes were made. The images or other third party material in this article are included in the article's Creative Commons license, unless indicated otherwise in a credit line to the material. If material is not included in the article's Creative Commons license and your intended use is not permitted by statutory regulation or exceeds the permitted use, you will need to obtain permission directly from the copyright holder. To view a copy of this license, visit http://creativecommons.org/licenses/by/4.0/.

(C) The Author(s) 2019 